\title{
Research on Air Defense Missile Target Assignment Algorithm
}

\author{
Ma Qi ${ }^{1, a}$, Han Jincheng ${ }^{2, b}$ \\ ${ }^{1}$ Department of scientific research, Air Defense Forces Academy,Zhengzhou, 450052, China \\ ${ }^{2}$ Department of scientific research, Air Defense Forces Academy,Zhengzhou, 450052, China \\ aemail: jsycjys@163.com, bemail:2657641275@163.com
}

\begin{abstract}
Keywords: Menace Estimate; Intercept Scheduling; Air Defense Missil; Fire Unit
\end{abstract}
\begin{abstract}
By analyzing the main factors of target threat degree, combined with existing interception sorting criteria, firstly considering the dynamic target case. Secondly, take the total loss as the objective function, establishs a decision-making model to intercept targets under higher specified targets without delay, gives the optimal algorithm of "weighted consistent loss decision problem". Finally, the example tells us that models and algorithms derived in this paper embodies the existing interception criteria, but also on the promotion of the existing guidelines, making the interception order more scientific and reasonable.
\end{abstract}

\section{Introduction}

When the air raid side concentrated assault on some protected objects, especially attacked in large-scale air attack formation, the air defense missile fire unit often face the target number of super saturation. Therefore, must divide the order of priority, and give priority to intercept the greatest threat value goal. As long as the target threat assessment criteria are reasonable, which can greatly improve the efficiency of air defense combat. Interception order, also called as intercept the priority queue, it will be put in a proper position as the interception order after the threat judgment. Interception order reflects the target threat degree for cover objects, but also reflects the emergency degree of intercepting target, through the target interception optimize allocation, so as to make the necessary preparations for the target interception ${ }^{[1]}$. Therefore, if the target interception order is reasonable, which will directly improve the level of operational effectiveness of air defense. This paper establishs mathematical model, and studys the dynamic target situation, then discusses the situation that is the specified target without delay, determines intercept target sequence of air defense missile fire unit, which makes the minimum total loss.

\section{Main Factors Affecting Threat Degree}

According to the factors of threat judgment process to consider, we constructed target threat assessment model, the various factors were compared, screening, extraction of relatively independent important factors, as elements to construct the threat estimation model. According to the current of air raid weapons, air raid style development and so on, we consider the following factors $^{[2]}$ :

(1) the target type. different types of air targets, the target feature, attack way, attack power and speed are different, thus there is stress on defending targets threat degree.

(2) the target speed. aerial target's flight speed directly influences on the killing probability of air defense missile. Even if the same types of targets, if the flight speed is different, they have different degree of threat. In general, the flight velocity of a target is larger, the level of threat is larger.

(3) the route shortcut. According to the shooting theory, the route shortcut of the air target is smaller, then the attack intention is more obvious, the degree of threat is greater.

(4) the height of the target. target flight height directly impact on the air defense missile position and target threat degree, generally speaking, the more highest, threat degree is smaller. 


\section{The Interception Sorting Problem}

With the development of air defense system, the domestic and foreign scholars study the interception sorting criterion, and propose many principles and method, these principles and methods is actually drawn according to the operational effectiveness, mainly include ${ }^{[2]}$ :

(1) the superior assigned priority to intercept the target, according to the higher order specified priority interception.

(2) the threat value big goals, priority to intercept.

(3) First come first interception on the same or similar target threat.

(4) when the emission region exists of many goals with the same or similar threat values, the first interception near.

This paper discusses when the specified targets are without delay, take the total loss as the objective function is researched and tries to provide a reasonable basis for intercepting decision.

\section{Dynamic interception model}

In the actual air raid and anti air combat process, there have been new air raid target into the launch area, the air defense missile fire unit continuously to receive the higher specified intercept target, having intercepted and no destructed aerial target is still in the emission region, some aerial targets into launch zone offen chang flight speed, so the number and distribution of the air target are in a constant state of change in emitting region. In this way, interception order of the air defense missile fire unit against air targets must also be constantly changing, in a certain time interval constantly updated, therefore, in the actual combat, interception order is a dynamic process of continuous change.

\section{The model assumptions}

(1) The targets are the same targets.

(2) After an interception, the command and control system can continue to turn the fire, and intercept the other target.

(3) In our defense weapon system sufficient conditions, all goals can be intercepted and destroyed.

(4) the preferred intercept goals assigned by superior will not be delayed.

(5) Attacking goals are dynamic target.

(6) the time of the target into the launch zone is consistent with the latest interception time.

\section{Model establishment}

Set operational period, attacking targets constitute objective set $X=\left\{X_{1}, X_{2}, \cdots, X_{n}\right\}$, the time of the target into the launch area are $r_{1}, r_{2}, \cdots, r_{n}$, the latest interception time respectively are $d_{1}, d_{2}, \cdots, d_{n}$, the time of the target into the launch zone is consistent with the latest interception time, that is when $r_{i} \leq r_{j}, d_{i} \leq d_{j}$. Superior assigned priority to intercept the target set $Y=\left\{X_{j_{1}}, X_{j_{2}}, \cdots, X_{j_{k}}\right\}, k \leq n$, and $Z=X / Y=\left\{X_{i_{1}}, X_{i_{2}}, \cdots, X_{i_{l}}\right\}, k+l=n$, the intercepting remaining time of Objectives $X_{j}(j=1,2, \cdots, n)$ is $d_{l s g j}(j=1,2, \cdots, n)$, and $s_{j}$ is the launching time of air defense missile intercepting the target $X_{j}, p_{j}$ is the actual time of intercepting target $X_{j}$, $C\{X\}$ represents interception total time of target set $X, U_{j}$ represents the unit loss, $c_{j}$ is the encounter time of missile and target $X_{j}$, meet $c_{j}=s_{j}+p_{j}$. Because of the target $X_{j}(j=1,2, \cdots, n)$ must be killed in the kill zone, so it must began to launch missile before $d_{l s g j}-p_{j}$, if $c_{j}>d_{l s g j}$, the target is not killed, it may bomb attacking, so the intercept delay occurred, caused a loss, denoted as 
$U_{j}=1$, if $c_{j} \leq d_{\text {lsgj }}$, then there is not the occurrence of intercept delay, denoted as $U_{j}=0$. Set $d_{m 1}$ is the flying speed of the first air targets; $d_{m z y 1}$ is the encounter time of the air defense missile and aerial target in the far field of kill zone; $d_{e}$ is a burst missile launching interval sum; $d_{p}$ is an aerial target shooting results evaluation time; $d_{z}$ is the firing transfer time. the problem is to determine a interception scheme, which makes the superior specified target without delay, as far as possible to kill more targets, caused minimum loss.

\section{Algorithm}

This paper consider the single target channel intercept dynamic multi-objective decision problem, if entering the time of emission region and the latest intercept time meet the consistency relation, so that the total loss minimizes, we put the problem referred to as dynamic loss decision

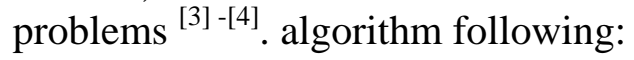

step1 At the moment of decision, the higher specified target set $Y=\left\{X_{j_{1}}, X_{j_{2}}, \cdots, X_{j_{k}}\right\}, k \leq n$, other target set need be intercepted $Z=X \backslash Y=\left\{X_{i_{1}}, X_{i_{2}}, \cdots, X_{i_{l}}\right\}, k+l=n$.

step2 the intercept remaining time of computing the higher specified target $X_{j_{i}}\left(X_{j_{i}} \in Y\right)$, and other target $X_{i_{l}}\left(X_{i_{l}} \in Z\right)$ are $d_{l s g j}(j=1,2, \cdots, n)$.

step3 Set $Z_{0}=Y, \quad X \backslash Z_{0}=\left\{X_{i_{1}}, X_{i_{2}}, \cdots, X_{i_{l}}\right\}$.

step4 scheduling from small to large in accordance with the shortest remaining time $d_{l s g j}$ in $Z_{0}$.

step5 if $k=l+1$, the algorithm terminate, $\left(Z_{l}, X \backslash Z_{l}\right)$ is the optimal sequence; if $k<l+1$, turns to step 6.

step6 Determine the order in accordance with the interception remaining time and two continuous follow-up targets conditions:

$$
\begin{aligned}
& \text { if } d_{l s g i_{k}} \geq d_{l s y i_{k-1}}+d_{m z y i_{k-1}}+2 d_{e}+d_{p}+d_{z} \text {, turns to step 6.1; } \\
& \text { if } d_{l s g i_{k}}<d_{l s y i_{k-1}}+d_{m z y i_{k-1}}+2 d_{e}+d_{p}+d_{z} \text {, turns to step 6.2. }
\end{aligned}
$$

step6.1 set $F_{k}=Z_{k-1} \cup\left\{X_{i_{k}}\right\}, Z_{k}$ is calculated as follows:

If there are no delays in the target $F_{k}$, set $Z_{k}=Z_{k-1} \cup\left\{X_{i_{k}}\right\}$; Otherwise,

(1) If the current is only one goal $X_{i_{r}} \in\left\{F_{k} \backslash Y\right\}$, such that for any $X_{i_{k}} \in\left\{F_{k} \backslash Y\right\}$, has $C\left\{F_{k} \backslash X_{i_{r}}\right\} \leq C\left\{F_{k} \backslash X_{i_{k}}\right\}$, then set $F_{k}=Z_{k} \backslash X_{i_{r}}$;

(2) more than one target $X_{i_{r}}$ satisfy condition $C\left\{F_{k} \backslash X_{i_{r}}\right\} \leq C\left\{F_{k} \backslash X_{i_{k}}\right\}$, then select the number of the largest target, before selecting this target to meet the conditions $C\left\{F_{k} \backslash X_{i_{r}}\right\} \leq C\left\{F_{k} \backslash X_{i_{k}}\right\}$, and the number of the smallest target as $X_{i_{r}}$, seting $Z_{k}=F_{k} \backslash\left\{X_{i_{r}}\right\}$ in $Z_{k}$, according to target interception remaining time according to the sort from small to large.

step6.2 Excluding the goal $X_{i_{k}}$, giving up interceptions.

step7 set $k=k+1$, turns to step 5, When a new target appears or not damage the original target, turns to step 1.

\section{Examples}

Suppose an air defense operations, there are five groups of target to enter (for convenience, the number of each batch is one, our antiaircraft missile fire units total have two goals channels, 
average speed of air defense missiles is $900 \mathrm{~m} / \mathrm{s}$, air raid targets related datas shown in Table 1 .

Table1 the air raid targets data

\begin{tabular}{ccccc}
\hline Target & Time into the launch area & Target distance & Target speed & Whether specified \\
\hline$X_{1}$ & $d=0 S$ & $106 \mathrm{~km}$ & $580 \mathrm{~m} / \mathrm{s}$ & no \\
$X_{2}$ & $d=0 S$ & $120 \mathrm{~km}$ & $580 \mathrm{~m} / \mathrm{s}$ & no \\
$X_{3}$ & $d=2 s$ & $110 \mathrm{~km}$ & $600 \mathrm{~m} / \mathrm{s}$ & no \\
$X_{4}$ & $d=2 s$ & $146 \mathrm{~km}$ & $600 \mathrm{~m} / \mathrm{s}$ & yes \\
$X_{5}$ & $t=3 s$ & $146 \mathrm{~km}$ & $600 \mathrm{~m} / \mathrm{s}$ & no \\
\hline
\end{tabular}

According to the algorithm, when $d=0$ s, no higher specified target, $Y=\phi$, other goals need to be intercepted are $Z=X \backslash Y=\left\{X_{1}, X_{2}\right\}$, intercept remaining time of calculating target $X_{1}, X_{2}$, and they are $d_{l s g 1}=71.6 s, d_{l s g 2}=81.1 \mathrm{~s}$, at this time the target channels are all idle, you can specify channel 1 and 2 to intercept the targets $X_{1}, X_{2}$, as $d=71.6 \mathrm{~s} 、 d=81.1 \mathrm{~s}$, damage target. the sum of the time interval is $4 s$ for the missile launchers, as $d=2 s$, goals $X_{3} 、 X_{4}$ enter the launch area, as $d=3 s$, goal $X_{5}$ enter the launch area, intercept remaining time are $d_{l s g 3}=73.3 s, d_{l s g 4}=97.3 s$, $d_{l s g 5}=97.3 \mathrm{~s}$. but the target $X_{4}$ is the specified target, must priority to be intercepted, so $d=4 s$, arrange for channels 1 to intercept the target $X_{4}$, as $d=4 \mathrm{~s}$, the intercept remaining time of $X_{3} 、 X_{5}$ are $d_{l s g 3}=72.5 s, d_{l s g 5}=93.3 \mathrm{~s}$, so arranges Channel 2 intercept target $X_{3}$, finally intercept targets $X_{5}$.

\section{Conclusion}

Based on the literature [4], we consider the case of dynamic objects, and findings of the literature [4] has be promoted. Secondly, take the total loss as objective function, establish a decision-making model on the condition of higher level specified target without delay, give the optimal algorithm for "consistent losses weighted decision problem", which can provide a theoretical reference for interception order.

\section{References}

[1] Xu Gao-ping, "Air defense missile fire units for air target interception scheduling problem”, Tactical Missile Technology China, vol. 6, Feb. 2007, pp. 01-09.

[2] Surface to air missiles firing command control model. Beijing: National Industry Press, 2009.

[3] Mao Ming, Yan Kewei, and Xiao Yi, “Target Assignment of Single Ship with Multi-way Ship-to-air Missile Weapon System in Air Defense”. Tactical Missile Technology China, vol. 4, Oct. 2009, pp. 18-20

[4] GaoJianjun, Guo Qiang. Air defense missile fire units intercept Ordination under the conditions of specified target without delay [J]. Journal of Air Defense Forces Academy, 2013(4): 53-54. 